\title{
TRANSFORMAÇÕES SOCIOECONÔMICAS E DE PRODUÇÃO DE UNIDADES DE PRODUÇÃO LEITEIRA (UPLS) DE UMA COOPERATIVA NA REGIÃO SUL DO RIO GRANDE DO SUL
}

\author{
ZANI, João Luíz ${ }^{1}$; \\ PETER, Cristina Mendes ${ }^{1}$; \\ KRUMMENAUER, Amanda ${ }^{1}$.
}

Recebido: 01/05/2019

Aceito: $26 / 08 / 2019$

${ }^{1}$ Faculdade de Veterinária, Universidade Federal de Pelotas.

\section{RESUMO}

\begin{abstract}
A produção leiteira é uma das atividades mais importantes do setor agropecuário no Brasil e no Rio Grande do Sul. Objetivou-se neste estudo realizar um levantamento socioeconômico e produtivo de Unidades de Produção Leiteira (UPLs) assistidas pela Cooperativa de Pequenos Produtores de Leite da Região Sul (COOPAL) no estado do Rio Grande do Sul, além de analisar suas transformações em um intervalo de 13 anos. Para isto, foram aplicados questionários a 188 produtores nos anos 2000 e 2013. Destes, 53,2\% (100) abandonaram a atividade durante o período. A maioria dos produtores não completou o primeiro grau, o número de analfabetos foi reduzido para zero, e houve aumento nos demais níveis de escolaridade. O volume diário de leite produzido por UPL variou positivamente. Houve incremento na qualidade e variedade dos alimentos ofertados aos animais, sendo as principais formas de oferta: pastagem de campo nativo, pastagem cultivada, milho e sal mineral. O resfriamento do leite de forma adequada à legislação aumentou, sendo utilizados principalmente tanques de imersão. O número de produtores com ordenhadeira mecânica aumentou de $9,09 \%$ para $48,86 \%$ ( $<<0,0001)$, porém a ordenha manual foi a principal utilizada. Houve um incremento no número de produtores que recebem algum tipo de assistência técnica, de 19 para 63 ( $p<0,0001)$. Acredita-se ter sido fundamental aos produtores que permaneceram na atividade a adaptação e adesão às tecnologias para atender ao aumento de exigências do mercado leiteiro.
\end{abstract}

Palavras-chave: Cadeia leiteira. Características produtor. Pequenos produtores. 


\section{INTRODUÇÃO}

Cerca de 150 milhões de propriedades rurais desenvolvem a produção leiteira em todo mundo (FAO, 2016). No Brasil, este é um dos setores mais importantes do agronegócio e consequentemente da economia nacional. A atividade leiteira está presente em aproximadamente 1,3 milhão de propriedades rurais, distribuídas em todo o país (SILVA et al., 2011). Estima-se que no ano de 2017, a produção de leite no Brasil tenha alcançado o volume de 33,49 bilhões de litros. Dados oficiais apontam que $8,5 \%$ dos estabelecimentos (cerca de 115.000 produtores) são responsáveis por $53,1 \%$ do leite produzido no país, ou seja, a grande maioria dos produtores de leite brasileiros $(91,5 \%)$ possuem rebanhos que produzem apenas $46,9 \%$ do volume total (IBGE, 2017a).

Dentre os Estados da federação, o Rio Grande do Sul é o segundo maior produtor de leite (IBGE, 2017a). De acordo com dados da Emater (2017), o número de produtores gaúchos supera os 170 mil, em 98,8\% dos municípios.

De acordo com a Organização das Nações Unidas para Alimentação e Agricultura (FAO, 2016), países em desenvolvimento, como o Brasil, têm como característica que a produção de leite é desenvolvida por pequenos agricultores, pois é uma atividade de retorno rápido e constante em todos os meses do ano. Os sistemas de produção leiteira no país são heterogêneos, devido à pluralidade cultural e econômica, por isso são necessários estudos regionais tanto dos sistemas de produção quanto de produção individuais, colaborando assim para o direcionamento, desenvolvimento e implementação de ações e políticas adequadas à realidade regional, visando a melhoria da produção e da qualidade do leite produzido.

Este estudo foi conduzido através do levantamento socioeconômico e produtivo de Unidades de Produção Leiteira (UPLs) atendidas pela Cooperativa de Pequenos Produtores de Leite da Região Sul (COOPAL), estado do Rio Grande do Sul, analisando as transformações em um intervalo de 13 anos. 


\section{MATERIAL E MÉTODOS}

Para o desenvolvimento do estudo, foram visitadas 188 Unidades Produtoras de Leite (UPLS) de associados da COOPAL/RS. A cooperativa contava, no início do estudo, com um quadro de 258 produtores de leite; assim, o estudo abrangeu $72,87 \%$ dos associados da cooperativa na primeira coleta de dados. Os locais visitados foram selecionados de acordo com a localização e o interesse dos produtores de participar do estudo. As propriedades estavam localizadas nos municípios Canguçu, São Lourenço do Sul, Morro Redondo e Piratini, pertencentes à região Sul do estado do Rio Grande do Sul.

Durante esta visita realizou-se a aplicação de um questionário estruturado, com questões abertas, dicotômicas ou de múltipla escolha, conforme constam no Quadro 1. Todas as propriedades foram visitadas nos anos de 2000 e 2013, sendo que, na segunda visita, os questionários foram aplicados somente àqueles produtores que ainda permaneciam realizando a atividade leiteira.

Quadro 1 - Relação de questões do questionário aplicado aos associados da COOPAL/RS nos anos 2000 e 2013, e tipo de variável para a formulação da resposta.

\begin{tabular}{|c|c|}
\hline Questão & Tipo \\
\hline “Até que série estudou?" & Aberta \\
\hline “Extrato de produção?” & $\begin{array}{l}\text { Múltipla escolha - até } 30 \text { Litros, } 30 \text { a } 50 \text { Litros, } 50 \text { a } 100 \\
\text { Litros e acima de } 100 \text { Litros }\end{array}$ \\
\hline “Número de hectares da propriedade?” & Aberta \\
\hline $\begin{array}{l}\text { "Número total de bovinos por categoria na } \\
\text { propriedade?" }\end{array}$ & Aberta \\
\hline $\begin{array}{l}\text { "Tipo de alimentação ofertada aos } \\
\text { bovinos?" }\end{array}$ & Aberta \\
\hline $\begin{array}{l}\text { "A propriedade conta com quais } \\
\text { instalações?" }\end{array}$ & $\begin{array}{l}\text { Múltipla escolha - Brete, Mangueira, Sala de ordenha, } \\
\text { Estábulo }\end{array}$ \\
\hline “Realiza resfriamento do leite?” & Dicotômica - Sim, não \\
\hline “Qual a forma de resfriamento do leite?" & Aberta \\
\hline $\begin{array}{l}\text { "De que forma é realizada a coleta do leite } \\
\text { (ordenha)?” }\end{array}$ & Aberta \\
\hline
\end{tabular}


O questionário e o termo de consentimento livre esclarecido (TCLE) foram aprovados pelo Comitê de Ética Humana da Comissão Nacional de Ética em Pesquisa - CONEP. As informações obtidas via questionários deram origem ao banco de dados armazenado no Microsoft Office Excel 2010 para posterior análise estatística das diferenças das médias nos dois períodos.

\section{RESULTADOS E DISCUSSÃO}

A amostra inicial do estudo, no ano 2000 , foi de 188 produtores de leite da região Sul do Rio Grande do Sul. Suas produções eram de caráter familiar, e os imóveis rurais possuíam dimensão média de 22,4 ha, se caracterizando como pequenas propriedades (área entre 1 e 4 módulos fiscais). O módulo fiscal dos municípios de Canguçu, São Lourenço do Sul e Morro Redondo é 16 ha e o de Piratini é 35 ha (INCRA, 2013).

Durante a segunda coleta de dados, em 2013, constatou-se que apenas 88 produtores permaneciam realizando a atividade. Deste modo, somente foram utilizados nas análises para avaliar as transformações ocorridas, os dados desses produtores.

De acordo com estimativas do Censo Agropecuário do IBGE (2017b), entre os anos de 2006 e 2017, houve uma redução em $13,30 \%$ no número de estabelecimentos produtores de leite. Em nosso estudo o índice de abandono foi de 53,2\%. Atribuímos influência a esta taxa por estarmos avaliando pequenas propriedades, com baixa escala de produção. Além disso, houve aumento da exigência sanitária pelo Ministério da Agricultura, Pecuária e Abastecimento, fator que gera a necessidade de maior capitalização para adequação às normas, o que acreditamos ser algo decisivo para os produtores que não estão recebendo o retorno financeiro esperado, deixarem a produção. De acordo com dados da Emater (2017), os produtores gaúchos apontam como suas principais dificuldades: falta de mão de obra, descontentamento em relação ao preço pago pelo leite, falta de perspectiva de sucessão familiar, reduzida escala de produção e deficiência na qualidade do leite. Já Ferrari et al. (2005), apontam como fatores que ameaçam a permanência no mercado, dos produtores de pequenos volumes de leite: a forma de pagamento pelo leite, que varia em função do volume produzido; o valor do frete e o acesso privilegiado dos produtores mais capitalizados às políticas públicas, como assistência técnica e crédito para financiamentos. 
Os dados obtidos neste estudo vão ao encontro dos de Montoya e Finamore (2010), que apontam que os extratos de produtores com áreas inferiores a 50 hectares foram os que mais expressaram o desejo de deixar a atividade nos próximos anos. Estima-se que até o ano de 2020, até 30 mil famílias gaúchas poderão abandonar a atividade leiteira, principalmente produções inferiores a 50 litros diários ou em áreas de difícil acesso (FETAG-RS, 2015).

De acordo com Picoli et al. (2014), técnicas de manejo inadequadas são praticadas com maior frequência por produtores que têm menor grau de instrução, portanto, os índices produtivos estão diretamente vinculados ao grau de conhecimento do produtor. Foi avaliado - grau de instrução dos produtores que permaneceram na atividade, conforme demonstrado na Tabela 1 . No ano 2000 , dois produtores eram analfabetos, enquanto em 2013 este número foi zero. Já o número de produtores que informaram como escolaridade: primeiro grau completo, segundo grau completo, ou pós-graduação, aumentou. Durante a primeira entrevista, realizada em 2000, 21 produtores não desejaram informar seu grau de escolaridade, enquanto no ano 2013 todos responderam.

Tabela 1 - Nível de instrução dos 88 produtores de leite da região Sul do Rio Grande do Sul, na área de abrangência da COOPAL, nos anos 2000 e 2013.

\begin{tabular}{llccccc}
\hline & Analfabeto & $\begin{array}{c}\text { 10 grau } \\
\text { incompleto }\end{array}$ & $\begin{array}{c}\text { 10 grau } \\
\text { completo }\end{array}$ & $\begin{array}{c}\text { 20 grau } \\
\text { completo }\end{array}$ & $\begin{array}{c}\text { Pós- } \\
\text { graduação }\end{array}$ & $\begin{array}{c}\text { Não } \\
\text { respondeu }\end{array}$ \\
\hline 2000 & $2(2,98 \%)$ & $63(94,03 \%)$ & $2(2,98 \%)$ & 0 & 0 & 21 \\
2013 & 0 & $81(92,04 \%)$ & $5(5,68 \%)$ & $1(1,14 \%)$ & $1(1,14 \%)$ & 0 \\
\hline
\end{tabular}

Em estudo nesta mesma região, Picoli et al. (2015) verificaram que a maior parte dos produtores de leite não havia completado o ensino fundamental $(67,9 \%), 4,2 \%$ eram analfabetos, $7,6 \%$ iniciaram o ensino médio, mas apenas $3 \%$ concluíram, e somente 1,1\% tinham ensino superior. Estes dados são semelhantes aos obtidos no Sul de Santa Catarina por Werncke et al. (2016), no qual 58\% dos produtores não possuíam nível fundamental completo, e aos de Picoli et al. (2017), em alguns municípios da região Sul do Rio Grande do 
Sul, que observaram a taxa de $64,15 \%$; entretanto, diferentemente, em ambos os anos das entrevistas, verificamos que a taxa dos produtores com primeiro grau incompleto foi superior a 90\%. Este índice pode estar relacionado a fatores geográficos e culturais ou, ainda, conforme os dados de Picoli et al. (2015), que indicam uma correlação positiva entre a escolaridade e o nível de adoção de tecnologias na produção, os produtores com menor grau de instrução, e deste modo, menor nível tecnológico, dependem exclusivamente de mão de obra familiar para o trabalho, contribuindo para que seus filhos deixem os estudos em idade precoce.

Na Tabela 2, são demonstrados os extratos de produção de leite nos anos 2000 e 2013. É possível verificar que aqueles produtores que permaneceram na atividade leiteira aumentaram sua produção, porque houve decréscimo no número de UPLs que produziam até 30 litros de leite/dia ( $p<0,0006)$, ao passo que ocorreu o aumento de todos os outros extratos.

Tabela 2 - Extrato de produção das 88 Unidades Produtoras de Leite (UPLs) localizadas nos Municípios da Região Sul do Rio Grande do Sul na área de abrangência da COOPAL, nos anos 2000 e 2013.

\begin{tabular}{ccccc}
\hline \multicolumn{5}{c}{ Extrato de Produção } \\
\hline Anos & Até 30L & $\mathbf{3 0 - 5 0 L}$ & $\mathbf{5 0 - 1 0 0 L}$ & $>\mathbf{1 0 0 L}$ \\
$\mathbf{2 0 0 0}$ & 66 & 12 & 9 & 1 \\
& $43^{*}$ & 21 & 17 & 7
\end{tabular}

(L): litros de leite; * Variação negativa no extrato de produção de até 30 litros por dia no período de 2000 a $2013(p<0,001)$.

Os dados apresentados concordam com as informações obtidas através da Pesquisa da Pecuária Municipal (IBGE, 2017a) referentes à produção nacional e estadual. No período entre 1996 e 2017, no Rio Grande do Sul, a média anual de produtividade por vaca ordenhada obteve uma variação positiva de $82 \%$, passando de 1805 litros/vaca/ano, para a média de 3284,6 litros/vaca/ano. Desta maneira, infere-se que a atividade na região seguiu a tendência, que é de crescimento da produção e da produtividade, com redução do número de propriedades rurais envolvidas (ZOCCAL, 2018). 
Montoya et al. (2014) informam que, na área de abrangência do Corede Sul (Conselhos Regionais de Desenvolvimento do Rio Grande do Sul), que engloba alguns dos municípios das propriedades analisadas, ocorreu um aumento da produção de leite entre os anos de 2001 e 2012, resultando numa variação de 13,3\%. Além disto, a produtividade por vaca também variou positivamente em $38 \%$, corroborando com os resultados apresentados neste trabalho. De acordo com os dados de Alves et al. (2012), 68\% do incremento da produção de leite nacional é explicado pela adoção de tecnologias, superando fatores como a elevação do trabalho (22\%) e expansão da área cultivada $(9,6 \%)$.

A alimentação ofertada aos animais determina a sustentabilidade econômica das UPLs, portanto, foram questionados quais os alimentos utilizados na dieta das vacas leiteiras. A nutrição é considerada o principal fator determinante do nível de produção de leite, influenciando diretamente a principal fonte de renda das fazendas leiteiras, e representa também o principal custo da atividade (PERES, 2001). De acordo com os dados computados na Tabela 3, em nosso estudo observou-se que, entre os anos de 2000 e 2013, houve um incremento no número de propriedades que ofertaram pastagem cultivada, assim como no número de propriedades que produziram silagem. $\mathrm{O}$ alimento concentrado, que no ano de 2000 era utilizado em apenas 20 (22,73\%) propriedades, passou a ser utilizado por 63 (71,59\%; $p<0,0001)$ no ano de 2013. Da mesma forma, a oferta de sal mineral variou positivamente, uma vez que o registro de seu uso foi de $42(47,73 \%)$ produtores no ano 2000, e de 74 (84,09\%; p<0,0001) em 2013. Conforme apontam Zanela et al. (2006), um dos principais problemas enfrentados nas UPLs da Região Sul do Rio Grande do Sul é a carência de alimentação adequada para seus rebanhos. 
Tabela 3 - Tipo de alimentação oferecida aos animais em 88 Unidades Produtoras de Leite (UPLs) na área de abrangência da COOPAL, nos anos de 2000 e 2013.

\begin{tabular}{|c|c|c|c|c|c|c|c|c|}
\hline \multicolumn{9}{|c|}{ Tipo de alimentação } \\
\hline & $\begin{array}{l}\text { Campo } \\
\text { nativo }\end{array}$ & $\begin{array}{l}\text { Pastagem } \\
\text { cultivada }\end{array}$ & Ração & Milho & $\begin{array}{c}\text { Sal } \\
\text { mineral }\end{array}$ & Silagem & Feno & Alfafa \\
\hline 2000 & $\begin{array}{c}72 \\
(81,82 \%)\end{array}$ & $\begin{array}{c}79 \\
(89,77 \%)\end{array}$ & $\begin{array}{c}20 \\
(22,73 \%)\end{array}$ & $\begin{array}{c}69 \\
(78,41 \%)\end{array}$ & $\begin{array}{c}42 \\
(47,73 \%)\end{array}$ & $\begin{array}{c}26 \\
(29,55 \%)\end{array}$ & $\begin{array}{c}3 \\
(3,41 \%)\end{array}$ & 0 \\
\hline 2013 & $\begin{array}{c}80 \\
(90,91 \%)\end{array}$ & $\begin{array}{c}82 \\
(93,18 \%)\end{array}$ & $\begin{array}{c}63^{*} \\
(71,59 \%)\end{array}$ & $\begin{array}{c}71 \\
(80,68 \%)\end{array}$ & $\begin{array}{c}74^{*} \\
(84,09 \%)\end{array}$ & $\begin{array}{c}32 \\
(36,36 \%)\end{array}$ & $\begin{array}{c}3 \\
(3,41 \%)\end{array}$ & $\begin{array}{c}1 \\
(1,14 \%)\end{array}$ \\
\hline
\end{tabular}

*Variação positiva no uso de ração e sal mineral para alimentação animal no período de 2000 a 2013 (p<0,001).

Em nosso registro, a variedade de alimentos aumentou, sendo uma das possíveis razões para o aumento significativo na produção total de leite. Os resultados sobre os tipos de alimentos são parcialmente semelhantes aos relatados por Ribeiro et al. (2015), que observaram, na região Sul do Rio Grande do Sul, que $80 \%$ das propriedades utilizavam campo nativo e ração; $60 \%$ utilizavam silagem e $40 \%$ faziam uso de outros diferentes alimentos.

A condição de armazenamento do leite após a ordenha nas propriedades rurais reflete diretamente na qualidade higiênico sanitária do leite. A legislação, atualmente determinada pelas Instruções Normativas no 76 e 77, proíbe a coleta de leite não refrigerado nas propriedades rurais no país (BRASIL, 2018). Verifica-se que, em consequência disso, desde o início das entrevistas, houve migração para sistemas de resfriamento.

Os dados referentes aos tipos de resfriamento do leite utilizados pelos produtores, nos anos de 2000 e 2013, estão demonstrados na Tabela 4. O resfriador era utilizado por 20 produtores em 2000, e 52 no ano de 2013, ou seja, um incremento de $160 \%$. O freezer foi utilizado por 20 produtores em ambos os anos, e o resfriamento em geladeira foi utilizado por 24 produtores em 2000 e 10 no ano de 2013, representando uma redução acima de 50\% na utilização deste método, onde não há controle de temperatura e consequente manutenção de padrão microbiológico.

Em pesquisa feita por Nero et al. (2009), a refrigeração do leite logo após a ordenha em tanques de imersão foi a prática mais utilizada por pequenos produtores, destinando o leite 
a tanques comunitários; procedimento permitido pela Instrução Normativa no 77, desde que o resfriamento ocorra em até 2 horas após a ordenha (BRASIL, 2018).

Tabela 4 - Tipos de resfriamento utilizados pelas 88 Unidades Produtoras de Leite (UPLS) na área de abrangência da COOPAL, nos anos de 2000 e 2013.

Tipo de resfriamento

\begin{tabular}{|c|c|c|c|c|c|c|c|c|}
\hline & Freezer & Geladeira & Água & $\begin{array}{c}\text { Tanque de } \\
\text { imersão }\end{array}$ & $\begin{array}{l}\text { Vidro } \\
\text { com } \\
\text { gelo }\end{array}$ & Gelo & $\begin{array}{l}\text { Tanque de } \\
\text { expansão }\end{array}$ & $\begin{array}{c}\text { Não } \\
\text { responderam }\end{array}$ \\
\hline 2000 & $\begin{array}{c}20 \\
(27,78 \%)\end{array}$ & $\begin{array}{c}25 \\
(34,72 \%)\end{array}$ & $\begin{array}{c}5 \\
(6,94 \%)\end{array}$ & $\begin{array}{c}20 \\
(27,78 \%)\end{array}$ & $\begin{array}{c}1 \\
(1,39 \%)\end{array}$ & $\begin{array}{c}1 \\
(1,39 \%)\end{array}$ & 0 & 16 \\
\hline 2013 & $\begin{array}{c}20 \\
(24,39 \%)\end{array}$ & $\begin{array}{c}10^{*} \\
(12,20 \%)\end{array}$ & 0 & $\begin{array}{c}44^{*} \\
(53,66 \%)\end{array}$ & 0 & 0 & $\begin{array}{c}8^{*} \\
(9,76 \%)\end{array}$ & 6 \\
\hline
\end{tabular}

*Variação do tipo de resfriamento comparando-se 2000 e 2013 ( $p<0,001)$.

Em ambas as entrevistas, respectivamente, $18,18 \%$ e 6,82\% dos 88 produtores optaram por não responder quanto à forma de resfriamento do leite. A legislação determina que as indústrias são proibidas de coletar leite não submetido ao resfriamento adequado, no entanto, cerca de $30 \%$ dos lácteos são comercializados no Brasil de maneira clandestina, sem industrialização e fiscalização sanitária (IBGE, 2017b). Assim, acreditamos que parte destes produtores não realizem o resfriamento do leite de maneira adequada, e escoem seus produtos através do mercado informal.

Verificou-se ainda que, dos 88 produtores no ano 2000, 8 possuíam ordenhadeira mecânica $(9,09 \%)$ e os 80 restantes realizavam a ordenha de forma manual $(90,91 \%)$. Já no ano de 2013, o número de produtores que utilizava a ordenha mecanizada como forma e obtenção do leite aumentou para 43 (48,86\%; $p<0,0001)$, ainda permanecendo 45 produtores fazendo uso da ordenha manual, ou seja, $51,14 \%$ dos produtores. Na região Sudeste do Rio Grande do Sul, Picoli et al. (2014) encontraram 39,3\% de prevalência de ordenha manual. Botega et al. (2008) relatam que existe insegurança por parte dos produtores na hora de investir em novas tecnologias, atribuída principalmente à preocupação com a capacidade de pagamento 
das parcelas e juros, visto que na maioria das vezes o capital para novos investimentos é oriundo de empréstimos bancários.

O acesso à assistência técnica também foi motivo de questionamento aos produtores durante a realização das entrevistas. Na primeira entrevista, 69 produtores relataram que não possuíam acesso à assistência técnica, seja pública ou privada, e apenas 19 recebiam visitas de um técnico. Em 2013, o número de produtores que não recebiam assistência decresceu para 25 ( $p<0,0001)$, ou seja, mais produtores obtiveram acesso à assistência técnica na produção de leite, passando para 63 produtores atendidos por técnicos. Embora não seja o único meio de acesso à informação, a escassez de assistência técnica diminui a probabilidade de adoção de novas práticas tecnológicas, principalmente nas médias e pequenas propriedades. Além disso, a orientação técnica, principalmente sobre programas de higiene e sanidade, mostra-se importante, influenciando positivamente em parâmetros de qualidade do leite (BAIRROS, 2009; MARTINELLI et al., 2014).

\section{CONCLUSÃO}

Conclui-se que ocorreram transformações nas UPLs da região Sul do estado do Rio Grande do Sul ao longo dos 13 anos entre a realização dos levantamentos socioeconômicos com a amostra de 188 produtores de leite. Foi observada a redução no número de produtores, uma vez que houve abandono da atividade em 53,2\% das propriedades avaliadas, porém com incremento no volume de leite produzido, com maior adoção de tecnologias de alimentação e ordenha, e maior adesão à assistência técnica. Apesar do menor número de produtores, isso não afetou o volume de produção como um todo, pois houve um incremento na adoção de tecnologia por parte daqueles que seguiram na atividade. Desta forma, acredita-se ter sido fundamental aos produtores que permaneceram na atividade a adaptação e adesão às tecnologias para atender ao aumento de exigências do mercado leiteiro. 


\section{SOCIOECONOMIC AND PRODUCTION TRANSFORMATIONS IN DAIRY PRODUCTION UNITS (DPUS) OF A COOPERATIVE IN THE SOUTH REGION OF RIO GRANDE DO SUL}

\section{ABSTRACT}

$\mathrm{M}$ ilk production is one of the most important activities of the agricultural sector in Brazil and Rio Grande do Sul. This study aimed to establish a socioeconomic and productive survey of dairy production units (DPUs) attended by the Cooperativa de Pequenos Produtores de Leite da Região Sul (COOPAL), in the state of Rio Grande do Sul, in addition to analyzing the transformations in a 13 year interval. For this, questionnaires were applied to 188 producers in the years 2000 and 2013. Of these, 53.2\% (100) abandoned the activity during the period. Most of the producers did not complete the first grade, the number of illiterates was reduced to zero, and there was an increase in other levels of schooling. The daily volume of milk produced by DPUs varied positively. The quality and variety of food offered to animals increased, with the main forms of food supply being: native field pasture, cultivated pasture, maize and mineral salt. The cooling of milk in an appropriate manner according to the legislation increased, being used mainly immersion tanks. The number of producers with mechanical milking machines increased from $9.09 \%$ to $48.86 \%$ ( $p<0.0001)$, but manual milking was the main used. There was an increase in the number of producers receiving some kind of technical assistance, from 19 to 63 ( $p<0.0001$ ). It is believed that it was fundamental to the producers who remained in the activity to adapt and adhere to the technologies to meet the increasing requirements of the dairy market.

Keywords: Dairy chain. Producer characteristics. Small producers.

\section{TRANSFORMACIONES SOCIOECONÓMICAS Y DE PRODUCCIÓN, EN EL INTERVALO DE 13 AÑOS, EN UNIDADES DE PRODUCCIÓN LECHERA (UPLS) DE UNA COOPERATIVA EN LA REGIÓN SUR DEL RÍO GRANDE DEL SUR}

\section{RESUMEN}

a producción lechera es una de las actividades más importantes del sector agropecuario en Brasil y en Rio Grande do Sul. El objetivo del estudio fue realizar un levantamiento - socioeconómico y productivo de unidades de producción lechera (UPLs) atendidas por la Cooperativa de Pequeños Productores de Leche da Región Sur (COOPAL), estado de Rio Grande do Sul, además de analizar las transformaciones en un intervalo de 13 años. Para ello, se aplicaron cuestionarios a 188 productores en los años 2000 y 2013 . De ellos, el 53,2\% (100) abandonó la actividad durante el período. La mayoría de los productores no 
completaron el primer grado, la cantidad de analfabetos fue reducido a cero, y hubo aumento en los demás niveles de escolaridad. El volumen diario de leche producido por UPL varió positivamente. La calidad y variedad de los alimentos ofrecidos a los animales aumentó, siendo las principales formas de oferta de alimentos: pastoreo de campo nativo, pastoreo cultivado, maíz y sal mineral. El enfriamiento de la leche de forma adecuada a la legislación aumentó, siendo utilizados principalmente tanques de inmersión. El número de productores con ordeñadora mecánica aumentó del 9,09\% al 48,86\% ( $p<0,0001)$, pero el ordeño manual fue la principal utilizada. Se ha producido un incremento en el número de productores que reciben algún tipo de asistencia técnica, de 19 a $63(p<0,0001)$. Se cree que fue fundamental para que los productores permanecieron en la actividad, la adaptación y adhesión a las tecnologías para atender al aumento de exigencias del mercado lechero.

Palabras clave: Cadena lechera. Características de productores. Pequeños productores.

\section{REFERÊNCIAS}

ALVES, E. R. A.; SOUZA, G. S.; ROCHA, D. P. Lucratividade da agricultura. Revista de Política Agrícola, v. 21, n. 2, p. 45-63, 2012.

BAIRROS, A. de. As transformações na cadeia produtiva do leite. 0 caso do Distrito São Bento, Carazinho, RS. Porto Alegre: UFRGS, 2009. 169p. Dissertação (Mestrado em Geografia), Programa de Pós-Graduação em Geografia, Instituto de Geociências, Universidade Federal do Rio Grande do Sul, 2009.

BRASIL. Ministério da Agricultura, Pecuária e Abastecimento. Instruções Normativas no 76 e no 77, de 26 de novembro de 2018. Diário Oficial da União, edição 230, seção 1, p. 9-10, 2018.

BOTEGA, J. V. L.; BRAGA JÚNIOR, R. A.; LOPES, M. A.; RABELO, G. F. Diagnóstico da automação na produção leiteira. Ciência e Agrotecnologia, Lavras, v. 32, n. 2, p. 635-639, 2008.

EMATER/RS - ASCAR. Relatório socioeconômico da cadeia produtiva do leite no Rio Grande do Sul: 2017. Porto Alegre: Emater/ASCAR, 2017. 64p. Disponível em: <http://biblioteca.emater.tche.br:8080/pergamumweb/vinculos/000006/00000679.pdf>. Acesso em: 04 abr. 2019.

FERRARI, D. L; MELLO, M. A.; TESTA, V. M.; SILVESTRO, M. L. Agricultores familiares, exclusão e desafios para inserção econômica na produção de leite em Santa Catarina. Informações Econômicas, v. 35, n. 1, p. 22-36, 2005. Disponível em:

<http://www.iea.sp.gov.br/ftpiea/ie/2005/tec2-0105.pdf> . Acesso em: 24 abr. 2019. 
FETAG-RS - FEDERAÇÃO DOS TRABALHADORES NA AGRICULTURA FAMILIAR DA REGIÃO SUL. Setor leiteiro adota censo e maior controle para superar crise. 2015. Disponível em: <https://gauchazh.clicrbs.com.br/economia/campo-e-lavoura/noticia/2015/03/setorleiteiro-adota-censo-e-maior-controle-para-superar-crise-4710305.html>. Acesso em: 26 de abr. 2019.

FAO - FOOD AND AGRICULTURE ORGANIZATION OF THE UNITED NATIONS. Dairy Production and Products - Milk Production. Disponível em: <http://www.fao.org/dairy-productionproducts/production/en/> . Acesso em: 24 abr. 2019.

IBGE - INSTITUTO BRASILEIRO DE GEOGRAFIA E ESTATÍSTICA. Pesquisa da Pecuária Municipal, 2017a. Disponível em:

<https://sidra.ibge.gov.br/pesquisa/ppm/quadros/brasil/2017> . Acesso em: 15 abr. 2019.

IBGE - INSTITUTO BRASILEIRO DE GEOGRAFIA E ESTATístICA. Censo Agropecuário, 2017b. Disponível em: <https://sidra.ibge.gov.br/> . Acesso em: 24 abr. 2019.

INCRA - INSTITUTO NACIONAL DE COLONIZAÇÃO E REFORMA AGRÁRIA. Sistema Nacional de Cadastro Rural - Índices Básicos de 2013. Disponível em:

$<$ http://www.incra.gov.br/sites/default/files/uploads/estrutura-fundiaria/regularizacaofundiaria/indices-cadastrais/indices_basicos_2013_por_municipio.pdf> . Acesso em: 19 mar. 2019.

MARTINELLI, R. R.; BÁNKUTI, F. I.; BÁNKUTI, S. M. S.; BRITO, M. M.; CASTRO, P. L. A influência da assistência técnica e extensão rural na qualidade do leite in natura. Revista Varia Scientia Agrárias, v. 4, n. 1, p. 9-22, 2014.

MONTOYA, M. A.; FINAMORE, E. B. Características dos produtores de leite do RS: uma análise a partir do Corede Nordeste. Indicadores Econômicos FEE, Porto Alegre, v. 37, n. 4, p. 213-224, 2010.

MONTOYA, M. A; PASQUAL, C. A.; FINAMORE, E. B. Panorama da produção leiteira no Rio Grande do Sul: perspectivas e gestão nas propriedades no Corede Produção. Passo Fundo: Ed. Universidade de Passo Fundo, 2014. 142p.

NERO, L. A.; VIÇOSA, G. N.; PEREIRA, F. E. Qualidade microbiológica do leite determinada por característica de produção. Ciência e Tecnologia de Alimentos, Campinas, v. 20, n. 2, p. 386390, 2009.

PERES, J. R. O leite como ferramenta do monitoramento nutricional. In: GONZALEZ, F. H. D.; DÜRR, J. W.; FONTANELE, R. (Eds.). Uso do leite para monitorar a nutrição e o metabolismo de vacas leiteiras. Porto Alegre: Biblioteca Setorial da FV - UFRGS, p. 29-43, 2001. 
PICOLI, T.; PETER, C. M.; BOESCHE, K. N.; FONSECA, R. N.; LOPES, M. G.; TEIXEIRA, A. G.; ZANI, J. L.; FISCHER, G. Estudo comparativo da produção leiteira em municípios da região sul do rio grande do sul. Science and Animal Health, v. 5, n. 2, p. 112-124, 2017.

PICOLI, T.; ZANI, J. L.; PETER, C. M.; LATOSINSKI, G. S.; FISCHER, G. Nível de instrução de produtores rurais e as características da produção leiteira. Science and Animal Health, v. 2, n. 2, p. 147-159, 2014.

PICOLI, T.; ZANI, J. L.; PETER, C. M.; ROLL, V. F. B.; RIBEIRO, M. E. R.; VARGAS, G. D.; HÜBNER, S. O.; LIMA, M.; FISCHER, G. Milk production characteristics in Southern Brazil. Semina:

Ciências Agrárias, Londrina, v. 36, n. 3, suplemento 1, p. 1991-1998, 2015.

RIBEIRO, M. E. R.; ZANELA, M. B.; ROSA, P. P.; JUCHEM, S. O.; ANTUNES, H. J. V.; DAVILA, A. S. Caracterização de Unidades de Produção Leiteira na Região Sul do Rio Grande do Sul. 1. Sistemas de Produção. In: CONGRESSO INTERNACIONAL DO LEITE, 13, 2015, Porto Alegre RS. ANAIS. Juiz de Fora - MG: Embrapa Gado de Leite, 2015.

SILVA, D. A. R.; OLIVO, C. J.; CAMPOS, B. C.; TEJKOWSKI, T. M.; MEINERZ, G. R.; SACCOL, A. G. F.; COSTA, S. T. Produção de leite de vacas da raça Holandesa de pequeno, médio e grande porte. Ciência Rural, Santa Maria, v. 41, n. 3, p. 501-506, 2011.

WERNCKE, D.; GABBI, A. M.; ABREU, A. S.; FELIPUS, N. C.; MACHADO, N. L.; CARDOSO, L. L.; SCHIMID, F. A.; ALESSIO, D. R. M.; FISCHER, V.; THALER NETO, A. Qualidade do leite e perfil das propriedades leiteiras no sul de Santa Catarina: abordagem multivariada. Arquivo Brasileiro de Medicina Veterinária e Zootecnia, v. 68, n. 2, p. 506-516, 2016.

ZANELA, M. B.; FISCHER, V.; RIBEIRO, M. E. R; STUMPF JÚNIOR, W.; ZANELA, C.; MARQUES, L. T.; MARTINS, P. R. G. Qualidade do leite em sistemas de produção na região Sul do Rio Grande do Sul. Pesquisa Agropecuária Brasileira. Brasília, v. 41, n. 1, p. 153-159, 2006.

ZOCCAL, R. Indicadores da produção mundial de leite. Anuário Leite EMBRAPA 2018, p. 1820, 2018. 